\title{
Denture Stomatitis An Underlying Menace
}

$\begin{array}{ll}\text { Pardeep Bansal }^{1} & \text { Rajesh Bhanot }^{3} \\ \text { Akshey Sharma }^{2} & \text { Gagan Chahal }^{4}\end{array}$

\begin{abstract}
Denture stomatitis is a yeast/fungal infection in the mouth caused by Candida, also known as thrush. It is not a type of infection that can be passed on to others. Denture wearers are the most common group to be affected, along with people who have difficulty in keeping their mouths clean. Diabetics and anyone who takes steroids by mouth or inhalers may also have problems with this condition. Some antibiotics are also responsible for causing thrush. If left untreated, the condition can cause soreness in the mouth and may lead to poorly fitting dentures in the future. So it is important to keep your mouth as clean as possible and rinse after every meal
\end{abstract}

Sharma

Keywords: Denture stomatitis, Candidiasis, Oral Thrush

\section{INTRODUCTION}

Denture stomatitis is a term describing the inflamed mucous membrane sometimes seen in the palate under a maxillary denture. It is essentially a multifactorial condition affecting the mucosa beneath the fitting denture surface. The etiologic factors include denture trauma, allergy, poor oral hygiene, $\mathrm{pH}$ level of saliva, age, sex, smoking, immune system deficiency and infection with Candida species. ${ }^{1}$ The mucosa becomes inflamed and red and the patient experiences soreness and burning sensation in the affected soft tissues. It is most frequently found under the upper complete denture.

Angular stomatitis (mild inflammation at the angles of the mouth) is frequently associated with denture stomatitis. This condition is typically caused by leakage of Candida infected saliva at the corners of the mouth. The sagging of facial tissues with age, collapsing of bite and immunosuppression also contribute to angular stomatitis. ${ }^{2}$

\section{PATHOPHYSIOLOGY}

Mucosal factors have been implicated in the etiology of this condition, as have behavioural and manner-of-use factors in patients who wear complete dentures. In these patients, the night time wear of the prosthetic appliance is the most significant factor. ${ }^{3}$

Although the dominant etiologic factor now appears to be fungal infection, other factors must be considered. These include the prosthetic device itself and also local and systemic factors in patients who are aging and edentulous. The extent of inflammation has been correlated with the presence of yeast colonizing the denture surface. ${ }^{4}$ Trauma has been shown to have a role in the production of basement membrane alterations involving expression of type IV collagen and laminin (alpha 1), thus indicating a possible relationship between these elements and denture stomatitis. ${ }^{5}$ Regarding the prosthesis-related factor, an allergy in the form of contact mucositis is suggested. This reaction may be related to the presence of resin monomers, hydroquinone peroxide, dimethyl-p -toluidine, or methacrylate in the denture. Furthermore, contact sensitivities such as denture stomatitis are more common with autopolymerising resins than with heat-polymerising denture-base materials.

Candida species have been identified in most patients or in all patients, ${ }^{6,7}$ with Candida albicans being the predominant species causing denture stomatitis. ${ }^{8}$ Whether the organism is merely commensal in this situation remains an issue because of the frequency of such organisms in the general population; the role of this organism as the sole etiologic factor in denture stomatitis is unclear. However, the presence of candidal organisms within the overall biofilm lends credence to its role in the development and maintenance of denture stomatitis. ${ }^{9}$ The etiology is best considered multifactorial, with the prosthesis considered the prime etiologic factor. The character of biofilm communities of denture wearers, however, has been shown to be distinctive when compared with healthy non - denturewearing individuals ${ }^{10}$

The disease is more common in elderly persons than in young persons because elderly persons are more likely to wear dentures and because their level of oral and denture hygiene is reduced. In addition, age-related chronic disease (eg, type II diabetes mellitus), iatrogenic drugs, and ageassociated immunocompromise contribute to this risk level.

\section{BIO FILM ATTACHMENT}

It is well known that any material placed in the mouth is rapidly exposed to an organic film of glycoproteins from the saliva. This forms a bio film on the surface of the denture material to which bacterial colonies and opportunistic organisms such as candida albicans attach.

Unpolished fitting surface of the denture is an ideal site 
for bio film formation and maturation particularly as the laboratory processing often leave small micro depressions on the fitting surface of the denture which provides sites for early attachment of bacteria. Additionally the absence of a rapid turnover of saliva over the fit surface helps the bacterial colonies to grow.

This bio film is difficult to eliminate completely and mechanical cleaning is unlikely to eliminate the entire bio film layer. Thus re- colonization occurs rapidly.

\section{HISTORY}

Denture stomatitis usually occurs in a patient who wears a complete maxillary denture or a partial denture. The presence of deteriorating temporary soft denture lining material and an improperly matched cleanser (which generally is the case) is associated with an increased presence of candidal organisms within the biofilm. ${ }^{11}$

In almost all patients, the duration of the lesion is usually unknown because of its asymptomatic nature. On rare occasions, patients may complain of slight bleeding and swelling in the involved area, as well as a burning sensation, a xerostomia-like quality, or cacogeusia. ${ }^{12}$

\section{PHYSICAL SIGNS AND SYMPTOMS}

Although symptoms are uncommon, the clinical presentation of intense erythema and edema in the part of the palatal mucosa covered by the denture base is a diagnostic finding (fig. 1).

At times, an obvious fungal infection in the form of white surface colonies or plaques may be observed on the mucosal surface. Variably intense erythema, which may also be associated with scattered petechiae, is distributed over the mucosa covered by the base of the denture but not beyond. Palpation of the involved mucosa reveals no tenderness or tissue friability. The severity of denture stomatitis can vary.

\section{STAGING}

The level of denture stomatitis can be classified as follows :

\section{Type I - Pinpoint hyperemia or localized simple inflammation (fig. 1)}

Type II - Diffuse erythema confined to the mucosa in contact with the denture base (fig. 2)

TypeIII - Granular surface or inflammatory papillary hyperplasia of the palate (fig. 3)

Type III denture stomatitis involves the epithelial response to chronic inflammatory stimulation secondary to yeast colonization and, possibly, low-grade local trauma resulting from an ill-fitting denture.

\section{MEDICAL CARE}

Despite the absence of symptoms, patients with advanced, chronic, or previously untreated cases must be treated because of the risk of IPEH (inflammatory papillary epithelial hyperplasia). IPEH usually needs to be surgically removed before the denture is emplaced or relined. In mild cases of IPEH, antifungal treatment without surgery might be an alternative before the dentures are relined or replaced.

\section{CURRENT TREATMENT MODALITIES}

The current treatment is divided into the treatment of acute condition and the tissue and the treatment of the denture.

1. Patient should be advised to take off their denture at night.

2. Good oral hygiene should be maintained and the mouth should be free of any periodontal disease. Chlorhexidine gluconate mouthwash helps in reducing plaque in the oral cavity.

3. The clinician should rule out the possibility that this reaction could be caused by improper design of the denture (which could cause unusual pressure on the mucosa), allergy to the denture base or inadequate curing of the denture base material. Identify and correct occlusal disharmonies, vertical dimension, and centric position.

4. The acute symptoms of the mucosa should be treated with Nystatin or Amphotericin B oral topical application.

- Nystatin pastilles (100,000 units): Denture stomatitis - to be dissolved slowly in the mouth 4 times daily for 7-14 days. Pastilles should not be chewed or swallowed whole. Alternatively nystatin oral drops 100,000 units/ml can be used. The preparation should be retained in the mouth for as long as possible before swallowing.

- Amphotericin B oral suspension $(100,000$ units $/ \mathrm{ml})$ : Denture stomatitis $-1 \mathrm{ml}(100 \mathrm{mg})$ rinse and hold in the mouth for as long as possible , 4 times daily for $10-15$ days.

- Miconazole gel ( $2 \%$ ): Angular stomatitis $-2.5 \mathrm{ml}$ (half a spoon) to be applied to the angles of the mouth for 10-14 days with Vit B complex supplements.

5. Disinfecting denture soak, tablets and rinses are useful in reducing and eliminating the bacterial load residing on the denture surfaces. ${ }^{13}$ Overnight soaking of denture in chlorhexidine gluconate solution $4 \%$ reduces microorganisms from the denture surface in 10 minutes but recolonization occurs easily. Sodium hypochlorite solution $1 \%$ helps in reducing the adhesiveness of the 
Candida in 10 minutes, but it bleaches the denture base materials. Hibatine $0.2-2 \%$ can also be used as a effective denture disinfectant. If the denture base contains metal, the patient should avoid using hypochlorite because it causes metal to tarnish. Another benefit of the regimen of overnight denture soaking is that the patients must remove their dentures for a prolonged period. Removal of the denture minimizes additional irritation and is a cornerstone of treatment.

6. Combining the soaking cleaning program with a twominute microwave treatment was overwhelmingly more effective to eliminate germs on the inside and outside of dentures worn from 12 days to 48 years, according to a recent study published in a 2003 issue of General Dentistry, the clinical, peer-reviewed journal of the Academy of General Dentistry (AGD). ${ }^{14}$ Only use this method for complete dentures. DO NOT use this method for dentures that contain metal, since no testing has been conducted on such appliances.

According to Dr. Glass, the combination of denture cleaners and microwave was especially effective to remove microorganisms (bacteria, yeasts and fungi) found in the depths of the dentures.

In cases that fail to respond to the usual treatments, consider the role of systemic disease and its impact on oral function and homeostasis. Chief among the systemic conditions that may affect denture stomatitis is type II diabetes mellitus. In patients with type II diabetes mellitus, the number of candidal organisms that adhere to the palatal epithelial cells is significantly increased; this finding supports the notion that this form of diabetes predisposes patients to Candida -associated denture stomatitis. However, a recent study group suggested that reduced resistance to candidal organisms present before the development of type II diabetes mellitus is related to denture stomatitis. ${ }^{15}$

Other conditions that may need to be excluded include cellular immunodeficiency and humoral immunologic disorders, HIV infection, hypothyroidism, poor diet, and iatrogenic drug use. ${ }^{16}$

\section{SURGICAL CARE}

IPEH should usually be surgically removed before the denture is relined.

\section{DETERRENCE/PREVENTION}

Denture sanitization is an important element in the treatment of denture stomatitis and should be emphasized to the patients. The 6-month incidence of denture stomatitis can be significantly reduced by educating nursing home caregivers about oral heath care.
The incidence of denture stomatitis and the duration of denture wear are highly correlated. Dentists can help prevent this condition by instructing patients to take their dentures out of their mouth for 6-8 hours each day.

Mechanical plaque control and appropriate denturewearing habits are the most important measures in preventing and treating the disease.

\section{PROGNOSIS}

In most patients, the elimination of mechanical and traumatic factors, the consistent use of oral hygiene measures, and the administration of local antimycotic therapy usually enables the inflammatory lesions to heal rapidly.

Recurrences are common when exacerbating factors are reintroduced.

\section{PATIENT EDUCATION}

The need for an educational component in a preventive oral health care program in geriatric institutions is unmet. ${ }^{17}$ Dental professionals who work with geriatric patients should address this need by implementing a preventive oral health care program. Such programs should include not only patient examinations and preventive care but also education for allied health care professionals and members of the patient's family.

Patients should be taught how to properly wear and sanitize their dentures and about how to perform good oral hygiene.
Fig. 1

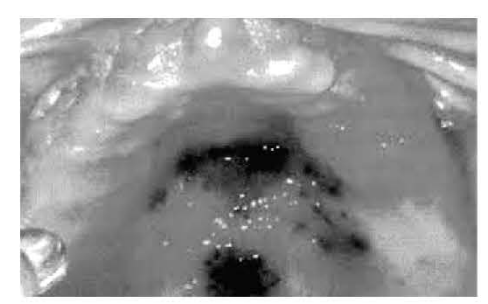

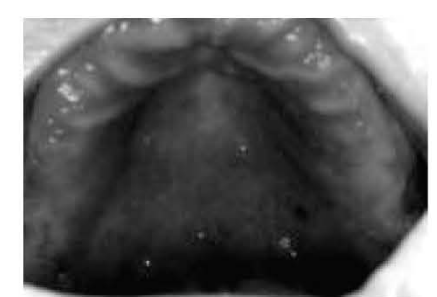

Fig. 2

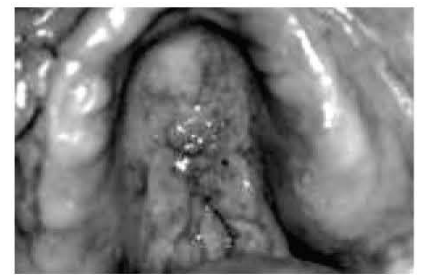

Fig. 3

\section{REFERENCES}

1. Newton AV, Denture sore mouth as possible etiology. Brit Dent J. 1962;112:357-60.

2. Cumming CG, Wight C, Blackwell CL, Wray D. Denture stomatitis in the elderly. Oral Microbiol Immunol. Apr 1990;5(2):82-5.

3. Fenlon MR, Sherriff M, Walter JD. Factors associated with the presence of denture related stomatitis in complete denture wearers: a preliminary investigation. Eur J Prosthodont Restor Dent. Dec 1998;6(4):145-7. 
4. Barbeau J, Seguin J, Goulet JP, et al. Reassessing the presence of Candida albicans in denture-related stomatitis. Oral Surg Oral Med Oral Pathol Oral Radiol Endod. Jan 2003;95(1):51-9.

5. Le Bars P, Piloquet P, Daniel A, Giumelli B. Immunohistochemical localization of type IV collagen and laminin (alpha1) in denture stomatitis. J Oral Pathol Med. Feb 2001;30(2):98-103.

6. Budtz-Jorgensen E, Stenderup A, Grabowski M. An epidemiologic study of yeasts in elderly denture wearers. Community Dent Oral Epidemiol. May 1975;3(3):115-9.

7. Cardash HS, Helft M, Shani A, Marshak B. Prevalence of Candida albicans in denture wearers in an Israeli geriatric hospital.Gerodontology. Winter 1989;8(4):101-7.

8. Marcos-Arias C, Vicente JL, Sahand IH, et al. Isolation of Candida dubliniensis in denture stomatitis. Arch Oral Biol. Feb 2009;54(2):127-31

9. Ramage G, Tomsett K, Wickes BL, Lopez-Ribot JL, Redding SW. Denture stomatitis: a role for Candida biofilms. Oral Surg Oral Med Oral Pathol Oral Radiol Endod. Jul 2004;98(1):53-9.

10. Campos MS, Marchini L, Bernardes LA, Paulino LC, Nobrega FG.
Biofilm microbial communities of denture stomatitis. Oral Microbiol Immunol. Oct 2008;23(5):419-24.

11. Nikawa H, Jin C, Makihira S, Egusa H, Hamada T, Kumagai H. Biofilm formation of Candida albicans on the surfaces of deteriorated soft denture lining materials caused by denture cleansers in vitro. $\mathrm{J}$ Oral Rehab. Mar 2003;30(3):243-50.

12. Dorocka-Bobkowska B, Budtz-Jorgensen E, Wloch S. Non-insulindependent diabetes mellitus as a risk factor for denture stomatitis. J Oral Pathol Med. Sep 1996; 25 (8):411-5.

13. Denture Hygiene In Geriatric Persons, The Internet Journal Of Geriatrics And Gerontology. 2010 Volume 6 Number 1.

14. Microwaves clean dentures best Public health, 10 Jun 2003

15. Vitkov L, Weitgasser R, Lugstein A, Noack MJ, Fuchs K, Krautgartner WD. Glycaemic disorders in denture stomatitis. J Oral Pathol Med. Oct 1999;28(9):406-9.

16. Wilson J. The aetiology, diagnosis and management of denture stomatitis. Brit Dent J. Oct 24 1998;185(8):380-4.

17. Matear DW. Demonstrating the need for oral health education in geriatric institutions. Probe. Mar-Apr 1999;33(2):66-71. 